\title{
On Summands Properties and Minkowski Subtraction
}

\author{
D. Borowska, H. Przybycień and R. Urbański
}

\begin{abstract}
In this paper we generalise the Sallee theorem from [J. Geom. 29 (1987)(1), 1-11, Theorem 4.3] into non-symmetric sets and give its proof in the terms of Minkowski subtraction.
\end{abstract}

Keywords. Minkowski subtraction, summands of convex sets

Mathematics Subject Classification (2000). Primary 52A07, secondary 26A27

\section{Preliminaries}

Let $X=(X, \tau)$ be a Hausdorff topological vector space and let $\mathcal{B}(X)$ be the family of all closed bounded and convex subsets of $X$. Let $\mathcal{K}(X)$ be the family of all compact members of $\mathcal{B}(X)$. For a subset $A \subset X$ of a vector space $X$ we denote by

$$
\operatorname{conv} A=\left\{x=\sum_{i=1}^{k} \alpha_{i} a_{i}: \alpha_{i} \geq 0, \sum_{i=1}^{k} \alpha_{i}=1, a_{i} \in A, k \in \mathbb{N}\right\}
$$

the convex hull of $A$ and by

$$
\text { aff } A=\left\{x=\sum_{i=1}^{k} \alpha_{i} a_{i}: \alpha_{i} \in \mathbb{R}, \sum_{i=1}^{k} \alpha_{i}=1, a_{i} \in A, k \in \mathbb{N}\right\} .
$$

the affine hull of $A$. The Minkowski sum for $A, B \in \mathcal{K}(\mathrm{X})$ is defined by

$$
A+B=\{a+b: a \in A, b \in B\} .
$$

We also define for $\lambda \in \mathbb{R}$ the sets $\lambda A=\{\lambda a: a \in A\}$ and $A-B=A+(-1) B$. We say that a set $B \in \mathcal{K}(X)$ is a summand of $A \in \mathcal{K}(X)$ if there exists a set $C \in \mathcal{K}(X)$ such that $B+C=A$.

D. Borowska, H. Przybycień and R. Urbański: Department of Mathematics and Computer Science, Adam Mickiewicz University, Poznań, Poland; dborow@amu.edu.pl, hubert@amu.edu.pl, rich@amu.edu.pl 
We introduce an equivalence relation " " on $\mathcal{K}^{2}(X)=\mathcal{K}(X) \times \mathcal{K}(X)$ by

$$
(A, B) \sim(C, D) \Longleftrightarrow A+D=B+C
$$

For a nonempty, compact, convex set $A \in \mathcal{K}(X)$ the support function $h(A, \cdot): X^{*} \rightarrow \mathbb{R}$ is given by

$$
h(A, x)=\max _{a \in A}\langle a, x\rangle,
$$

where $\langle\cdot, \cdot\rangle$ is the dual pairing between $X$ and $X^{*}, X^{*}$ is the dual space (see [4]). Let

$$
A-B=\{x \in X: x+B \subset A\}
$$

be the Minkowski subtraction of $A$ and $B$. The equality

$$
A-B=\bigcap_{b \in B}(A-b)
$$

holds true (see [2]). For $A, B, C \in \mathcal{K}(X)$ the following conditions hold:

(i) $A+(B \dot{-} A) \subset B$.

(ii) If $A=B+C$, then $B=A-C$.

(iii) If $B \subset C$, then $A-C \subset A-B$.

(iv) If $\alpha \in \mathbb{R}$, then $\alpha(A \dot{-} B)=\alpha A \dot{-} \alpha B$.

(v) If $\alpha, \beta \in \mathbb{R}, \alpha \geq \beta$, then $\alpha A \dot{-} \beta A=(\alpha-\beta) A$.

(vi) If $B+C \subset A$, then $B \subset A \dot{-} C$.

For more properties of Minkowski subtraction see [2].

If aff $A \cap$ aff $B=\{p\}$, then we write $A \oplus B$ instead $A+B$ and we call it a direct sum of $A$ and $B$. If $a_{1}, \ldots, a_{n+1}$ are $n+1$ points affinelly independent, then the set conv $\left\{a_{1}, \ldots, a_{n+1}\right\}$ we call the $n$-simplex. By the cube in the $\mathbb{R}^{n}$ we mean a cartesian product $\left[a_{1}, b_{1}\right] \times \cdots \times\left[a_{n}, b_{n}\right]$ of intervals, where $a_{k} \leq b_{k}$ for $k=1, \ldots, n$. Let $f \in X^{*}, A \subset f^{-1}(0)$ and $B \subset f^{-1}(0)+x$ for some $x \in X$, then the set $\operatorname{conv}(A \cup B)$ we call a frustum with the bases $A$ and $B$. We call the set $A \in \mathcal{K}(X)$ centrally symmetric if $A=-A$.

\section{Introduction}

In this paper we focus on the summands properties. We generalise the Sallee theorem from [5, Theorem 4.3] into non-symmetric sets and give its proof in the terms of Minkowski subtraction. We also introduce the operator $D^{n}$, prove some of its basic properties and show its connections with the operator $\Omega^{n}$ defined by Sallee for symmetric sets in $\mathbb{R}^{n}$ ([5, Theorems 3.1 and 3.2]). We use this connections to the modification of the proof of Theorem 4.3 given in [5]. By the 
way, we receive some new properties of Minkowski subtraction. Then, we give some examples of members of the family $\mathcal{F}$ for which the implication

$$
C \dot{-} A=B \text { and } C \dot{-} B=A \quad \Rightarrow \quad A+B=C
$$

is not true. This problem was considered by Sallee in [5]. In the last chapter we investigate the following problem: for which sets $A \in \mathcal{K}(X)$ the set $A-B$ is a summand of the set $A$. Although it is quite a complicated issue and we do not receive a full characterisation of that family, now we are able to define the family $\mathcal{C}$ whose each element has this property. That family is rather vast. This problem was solved in the space $\mathbb{R}^{2}$ (see [7]).

First let us define an operator

$$
\begin{aligned}
& D^{k}: \mathcal{K}(X) \times \mathcal{K}(X) \rightarrow \mathcal{K}(X) \cup\{\emptyset\}, \quad k \in \mathbb{N} \\
& D^{k}(B, A)= \begin{cases}B \dot{-} A & \text { for } k=1 \\
D^{1}\left(B, D^{k-1}(B, A)\right) & \text { for } k>1 .\end{cases}
\end{aligned}
$$

In [5] Sallee introduced the following operator:

$$
\begin{aligned}
& \Omega^{k}: \mathcal{K}\left(\mathbb{R}^{n}\right) \times \mathcal{K}\left(\mathbb{R}^{n}\right) \rightarrow \mathcal{K}\left(\mathbb{R}^{n}\right), k \in \mathbb{N} \\
& \Omega^{k}(B, A)= \begin{cases}B & \text { for } k=0 \\
\bigcap\left\{x+A: x \in \Omega^{k-1}(B, A)\right\} & \text { for } k>0,\end{cases}
\end{aligned}
$$

where $A$ is a centrally symmetric set in $\mathbb{R}^{n}$.

\section{The operators $D^{n}$ and $\Omega^{n}$}

In this section we give some basic properties of the operations $D^{n}$ and $\Omega^{n}$.

Theorem 3.1. Let $A, B \in \mathcal{K}(X)$. Then $D^{3}(A, B)=D^{1}(A, B)$.

Proof. For $A, B, C, M, N \in \mathcal{K}(X)$ the following implication is true:

$$
M \subset N \Rightarrow A \dot{-} N \subset A \dot{-} M .
$$

Since $B+(A \stackrel{-}{-}) \subset A$ we obtain

$$
B \subset A \dot{-}(A \dot{-} B)
$$

Replacing $B$ by $A \dot{-} B$ in the above inclusion we get $A-B \subset A \dot{-}(A \dot{-}(A \dot{-} B))$ which is equivalent to the following expression $D^{1}(A, B) \subset D^{3}(A, B)$. On the other hand, putting $M=B, N=A \dot{-}(A \dot{-} B)$ and applying the equalities (2) and (1) we obtain $A \dot{-}(A \dot{-}(A \dot{-} B)) \subset A \dot{-} B$ which proved $D^{3}(A, B) \subset$ $D^{1}(A, B)$. 
Theorem 3.2. Let $A, B \in \mathcal{K}(X)$ and $B$ be a centrally symmetric set. Then the following equation holds true:

$$
\Omega^{n}(A, B)=D^{n}\left(B,(-1)^{n} A\right) \quad \text { for } n \in \mathbb{N} .
$$

Proof. From the definition of Minkowski subtraction we have

$$
\Omega^{1}(A, B)=\bigcap_{x \in A}(B-(-x))=\bigcap_{y \in-A}(B-y)=B \dot{-}(-A)=D^{1}(B,-A) .
$$

We shall prove the equality $\Omega^{1}(A, B)=-(B \dot{-} A)$. From the definition of the subtraction we have $-\{y: y+A \subset B\}=-\{y:-y-A \subset-B\}=-\{y:-y-$ $A \subset B\}=\{-y:-y-A \subset B\}=\{y: y-A \subset B\}=B \dot{-}(-A)=D^{1}(B,-A)$. Hence

$$
\Omega^{2}(A, B)=\Omega^{1}\left(\Omega^{1}(A, B), B\right)=\Omega^{1}\left(D^{1}(B,-A), B\right)=D^{1}\left(B,-D^{1}(B,-A)\right) .
$$

It is easy to see that

$$
D^{1}(B,-A)=B \dot{-}(-A)=-(B \dot{-} A)=-(B \dot{-} A)=-D^{1}(B, A) .
$$

Using the equalities (3) and (4) we obtain $\Omega^{2}(A, B)=D^{1}\left(B,\left(D^{1}(B, A)\right)\right)=$ $D^{2}(B, A)$. From the definition of $\Omega^{n}$ we have $\Omega^{2}(A, B)=\Omega^{1}\left(\Omega^{1}(A, B), B\right)=$ $B \dot{-}[-(B \dot{-}(-A))]=B \dot{-}(B \dot{-} A)$.

In [5] was proved that $\Omega^{3}(A, B)=\Omega^{1}(A, B)$ for $A, B \subset \mathbb{R}^{n}$. Analogously it can be proved that $\Omega^{3}(A, B)=\Omega^{1}(A, B)$ for $A, B \subset X$. From the definition of $\Omega^{n}$, Theorem 3.1 and the above we have $\Omega^{3}(A, B)=\Omega^{1}\left(\Omega^{2}(A, B), B\right)=$ $\Omega^{1}\left(D^{2}(A, B), B\right)=D^{1}\left(B,-D^{2}(B, A)\right)=-D^{1}\left(B, D^{2}(B, A)\right)=-D^{3}(B, A)=$ $D^{3}(B,-A)=-D^{1}(B, A)=D^{1}(B,-A)=\Omega^{1}(A, B)$.

Now, using the Theorem 3.1 we obtain that $\Omega^{n}(A, B)=D^{n}\left(B,(-1)^{n} A\right)$ for $n \in \mathbb{N}$.

Corollary 3.3. Let $A, B \in \mathcal{K}(X)$. Then

$$
D^{n}(B, A)= \begin{cases}B \dot{-} A & \text { for odd } n \\ B \dot{-}(B \dot{-} A) & \text { for even } n\end{cases}
$$

Moreover, if $B$ is a centrally symmetric set, then

$$
\Omega^{n}(A, B)= \begin{cases}B \dot{-}(-A) & \text { for odd } n \\ B \dot{-}(B \dot{-} A) & \text { for even } n\end{cases}
$$

Definition 3.4. Let $X$ be a topological vector space and let $A, B, M \in \mathcal{K}(X)$. A pair $(A, B)$ is called an $M$-pair if and only if $A+B=M$. 
Definition 3.5. Let $X$ be a topological vector space. A pair of sets $(A, B) \in$ $\mathcal{K}^{2}(X)$ is called the pair of sets of constant width relative to $S$ or $S$-pair if $A-B=\lambda$ for some $\lambda>0$, where $S$ is a centrally symmetric subset of $X$.

Notice, that $S$-pair and $M$-pair are different.

Theorem 3.6. Let $X$ be a locally convex topological vector space and let $A, B \in$ $\mathcal{K}(X)$. If $(A, B)$ is an $S$-pair and there exists $(C, D)$ such that $(A, B) \sim(C, D)$ then $C-D$ is centrally symmetric.

Proof. Using the quivalence

$$
h(A, u)+h(B,-u)=\lambda h(S, u) \Longleftrightarrow A-B=\lambda S
$$

and the equivalence relation

$$
(A, B) \sim(C, D) \Longleftrightarrow A+D=B+C
$$

we obtain

$$
\begin{aligned}
h(A, u)+h(D, u) & =h(B, u)+h(C, u) \\
h(B,-u)+h(C,-u) & =h(A,-u)+h(D,-u) .
\end{aligned}
$$

Hence from the above equality

$$
\lambda h(S, u)+h(D, u)+h(C,-u)=\lambda h(S,-u)+h(C, u)+h(D,-u) .
$$

Therefore $h(C, u)+h(D,-u)=h(D, u)+h(C,-u)$. It is easy to see that $h(D,-u)=\max \langle D,-u\rangle=\max \langle-D, u\rangle=h(-D, u)$, hence from the above equality we obtain $h(C, u)+h(-D, u)=h(D, u)+h(-C, u), h(C-D, u)=$ $h(D-C, u)$. Therefore $C-D=D-C$.

Definition 3.7. Let $X$ be a normed space and $A \in \mathcal{K}(X)$. We call the set $A$ a set of constant width if $A-A=\lambda B$, where $B$ is the unit ball.

Definition 3.8. Let $X$ be a topological vector space and $A \in \mathcal{K}(X)$. We call the set $A$ a set of constant $S$-width if $A-A=\lambda S$, where $S$ is a centrally symmetric subset of $X$.

Definition 3.9. Let $X$ be a normed space. A pair $(A, C) \in \mathcal{K}^{2}(X)$ is called the pair of sets of constant width if $A-C=\lambda B$, where $B$ is a unit ball.

Theorem 3.10. Let $X$ be a normed space and let $A, B, M \in \mathcal{K}(X)$. Then the following statements are equivalent:

(i) $A$ is a summand of $M$;

(ii) $\left(A,-D^{1}(M, A)\right)$ is an $M$-pair. 
Proof. If $A$ is a summand of $M$, then there exists $B \in \mathcal{K}(X)$ such that $A+$ $B=M$. Hence $A+D^{1}(M, A)=M, B=M \stackrel{\bullet}{-} A=D^{1}(M, A)$. We have $-B=-D^{1}(M, A)$.

Conversely, assume that $\left(A,-D^{1}(M, A)\right)$ is an $M$-pair. By the definition of an $M$-pair, $A$ is a summand of $M$.

For $A, B \in \mathcal{K}(X)$ we will use the notation $A \vee B=\operatorname{conv}(A \cup B)$. For two elements $a, b \in \mathcal{K}(X)$ the interval with end points $a$ and $b$ will be denoted by $[a, b]=a \vee b$.

Example 3.11. Let $K=a \vee b \vee c$ and $K^{\prime}=-2 K$, where $K \subset \mathbb{R}^{2}$ is the Reuleaux triangle (see [1],[6]). Then $K$ and $K^{\prime}$ are the sets of constant width. Notice that $-2 K \dot{-} K$ is not a set of constant width (see Figure 1 ).

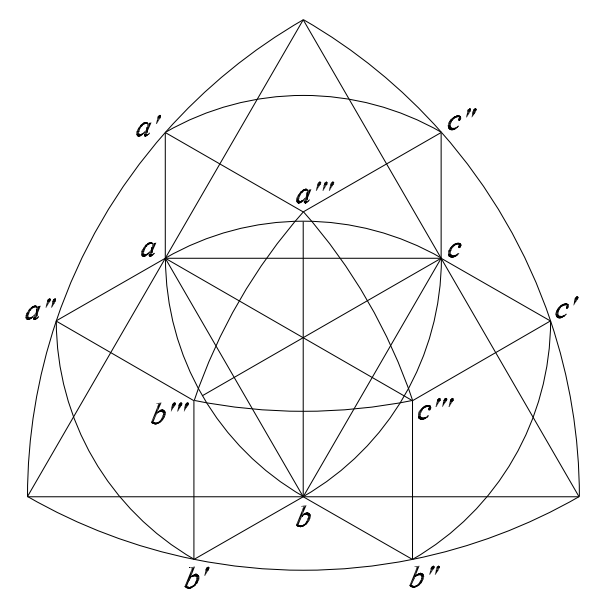

Figure 1: Reuleaux triangles

It is easy to observe that the set $-2 K \dot{-} K$ is not a triangle. Its boundary is the union of three arcs with the vertices $a^{\prime \prime \prime}, b^{\prime \prime \prime}, c^{\prime \prime \prime}$. The curvature of every of those arcs is less than $\left\|a^{\prime \prime \prime}-b^{\prime \prime \prime}\right\|^{-1}$. So $-2 K \dot{-} K$ is not a set of constant width.

Let $K=a \vee b \vee c \vee d \vee e$ be a pentagon of constant width and let $\alpha<-1$. Similary we can show that $\alpha K \dot{-} K$ is not a set of constant width (see Figure 2).

Corollary 3.12. The Minkowski subtraction does not preserve the constant width of sets.

Proposition 3.13. The result of Minkowski subtraction of two centrally symmetric sets is centrally symmetric set.

Proof. Let $A=-A, B=-B$. Then $-(A \dot{-} B)=(-1) A \dot{-}(-1) B=A \dot{-} B$. 


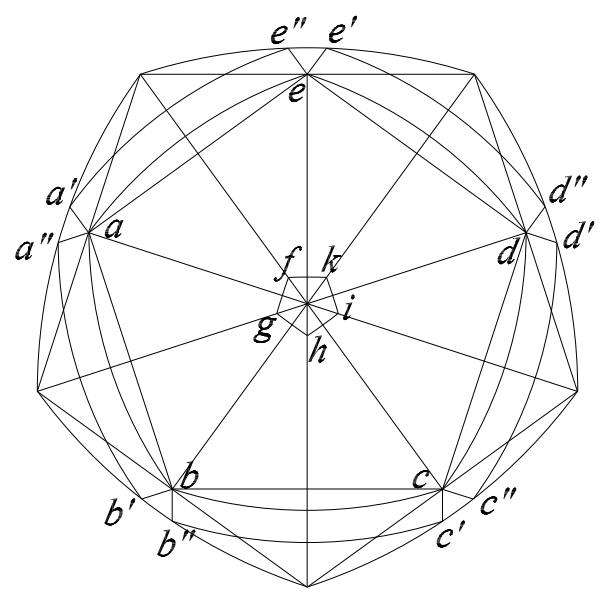

Figure 2: Reuleaux pentagons

If $A, B, S \in \mathcal{K}(X)$ and $A+B=S$, then $S \dot{-} A=B$ and $S \dot{-} B=A$. We define a class $\mathcal{S}$ of sets $S \in \mathcal{K}(X)$ such that $S-A=B$ and $S-B=A$ imply $A+B=S$. Moreover let us define $\mathcal{F}=\mathcal{K}(X) \backslash \mathcal{S}$. It is easy to observe that $S \in \mathcal{S}$ if and only if the equality $S \dot{-}(S-A)=A$ implies that $A$ is a summand of $S$.

Example 3.14. G. T. Sallee in [5] gives the following example (see Figure 3) of the member of the family $\mathcal{F}$.

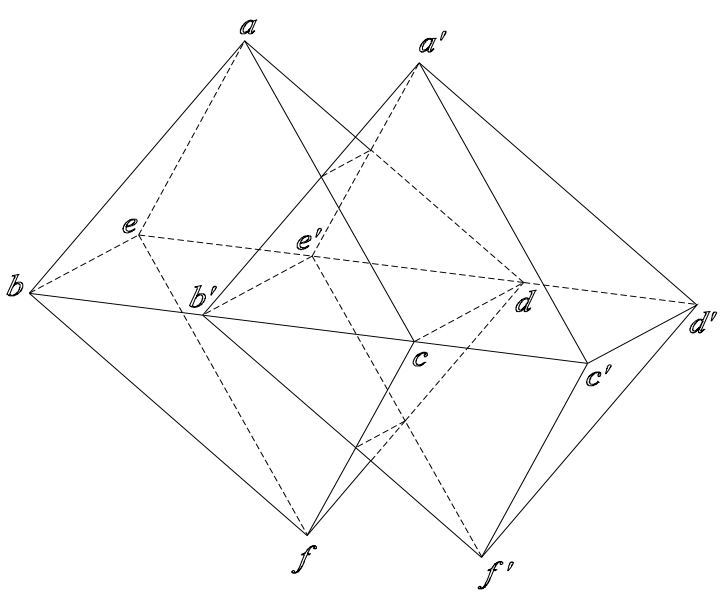

Figure 3: Octahedron

Let $M$ be the octahedron, $B=b \vee b^{\prime}, A$ is the eight faceted set. Then 
$A=M \dot{-} B, B=M \dot{-} A, A+B \neq M:$

$$
\begin{aligned}
A & =c \vee c^{\prime} \\
M & =a \vee i \vee j \vee c^{\prime} \vee d^{\prime} \vee e^{\prime} \vee f^{\prime} \vee g^{\prime} \vee b^{\prime} \\
B & =h \vee c \vee c^{\prime} \vee d^{\prime} \vee e^{\prime} \vee k^{\prime} \vee k \vee e \vee d .
\end{aligned}
$$

Let the polytope $A$ be the convex union of a hexagonal pyramid, a hexagonal prism and a wedge (see Figure 4). One of possible intersections $A \cap(A-x)$ is the convex union $B$ of four-sided pyramid and four-sided prism, which is not a summand of $A$. From the construction of the above set we obtain a quite wide class of subsets of $\mathbb{R}^{3}$ which belongs to the family $\mathcal{F}$.

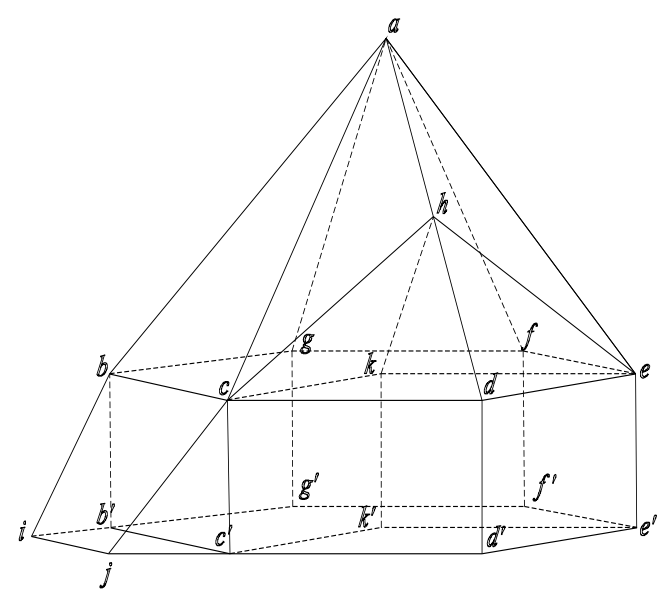

Figure 4: A polytope

The problem can be formulated as follows: to find all the sets $S$, for which the equality $S \dot{-}(S \dot{-} A)=A$ implies that $A$ is a summand of $S$.

\section{Some properties of Minkowski subtraction}

In this section we give some properties of Minkowski subtraction and prove the Sallee theorem in its terms.

Theorem 4.1. Let $A, B \in \mathcal{K}(X)$ and let $L$ be a linear invertible transformation in $X$. Then $L A \dot{-} L B=L(A \dot{-} B)$.

Proof. Let $y \in L(A-B)$. Then there exists $x \in X$ such that $y=L x$ and $x+B \subset A$. So $y+L B=L x+L B \subset L A$. Hence $y \in L A \dot{-} L B$ and $L(A \dot{-} B) \subset L A \dot{-} L B$. If $L$ is invertible then using the above inclusion we get $L^{-1}(L A \dot{-} L B) \subset A \dot{-} B$. Therefore $L A \dot{-} L B \subset L(A \dot{-} B)$. 
Example 4.2. Let $L: \mathbb{R}^{2} \rightarrow \mathbb{R}^{2}, L(x, y)=(x, 0)$ and $A=[0,1] \times[0,1]$, $B=[0,1] \times[0,2]$, then $L(A \dot{-} B=\emptyset$ and $L A \dot{-} L B=\{(0,0)\}$. So the assumption of invertibility of $L$ is essential.

Corollary 4.3. Let $L$ be a linear invertible transformation in $X$. If $L(A)=A$ and $L(B)=B$, then $L(A-B)=A-B$.

Corollary 4.4. If $L$ is a linear invertible transformation in $X, L: X \rightarrow X$ and $S \in \mathcal{S}$, then $L(S) \in \mathcal{S}$.

Proof. Assume that $L(S) \dot{-}(L(S) \dot{-} A)=A$. Then

$$
L^{-1}(L(S) \dot{-}(L(S) \dot{-} A))=L^{-1}(A) .
$$

Now by the linearity of $L$ and $L^{-1}$ we have $L^{-1}\left(L(S)-L^{-1}(L(S)-A)\right)=$ $L^{-1}(A)$. Hence we obtain $S \dot{-}\left(L^{-1}(L(S)) \dot{-} L^{-1}(A)\right)=L^{-1}(A)$ and $S \dot{-}$ $\left(S-L^{-1}(A)\right)=L^{-1}(A)$. Putting $L^{-1}(A)=B$ we have $S \dot{-}(S \dot{-} B)=B$. Since $S \in \mathcal{S}$ so $B$ is a summand of $S$. Therefore by the definition of the summand there exists a set $C \in \mathcal{K}(X)$ such that $B+C=S$. Putting $L^{-1}(A)+C=S$ and from properties of the operator $L$ we obtain

$$
L L^{-1}(A)+L(C)=L(S),
$$

hence $A+L(C)=L(S)$. Then $A$ is a summand of $L(S)$ and $L(S) \in \mathcal{S}$.

Lemma 4.5. Let $A, B, C \in \mathcal{K}(X)$ and aff $A=$ aff $B$. Let moreover $C \subset W$, where $W$ is a linear space, such that $W \cap$ aff $A=\{p\}$. Then

$$
\begin{aligned}
(A \oplus C) \cap(B \oplus C) & =(A \cap B) \oplus C \\
(A \oplus C)+B & =(A+B) \oplus C .
\end{aligned}
$$

Proof. We assume $V=\operatorname{aff} A=\operatorname{aff} B$ and $W$ is a linear space with $W \cap V=$ $\{p\}$. Let $U=V \oplus W$. Let us immerse isomorphicaly the space $V \oplus W$ into $V \times W \backsim V \oplus W \rightarrow V \times W$. We denote

$$
\begin{aligned}
& \widetilde{A}=\{(x, 0): x \in A\} \subset V \times W \\
& \widetilde{B}=\{(y, 0): y \in B\} \subset V \times W \\
& \widetilde{C}=\{(z, 0): z \in C\} \subset V \times W .
\end{aligned}
$$

We have $(\widetilde{A}+\widetilde{C}) \cap(\widetilde{B}+\widetilde{C})=\{(x, z): x \in A, z \in C \cap\{(y, z): y \in B$, $z \in C\}=\{(u, z): u \in A \cap B, z \in C\}=\widetilde{A} \cap \widetilde{B}+\widetilde{C}$ since the isomorphic immersion is 1-1. So we have $(A \oplus C) \cap(B \oplus C)=A \cap B \oplus C$. To prove the second equality let us observe that aff $(A+B) \subset$ aff $A+$ aff $B=2$ aff $A$. Hence aff $(A+B) \cap$ aff $C \subset 2$ aff $A \cap$ aff $W=2$ aff $A \cap 2 W=2($ aff $A \cap W)=2\{p\}$. 
Corollary 4.6. Let $A_{t}, A_{t_{1}}, A_{t_{2}}, C \subset X$ for $t, t_{1}, t_{2} \in T$ and aff $A_{t_{1}}=$ aff $A_{t_{2}}$. Moreover let $C \subset W$, where $W$ is an affine subspace of $X$, such that $W \cap$ aff $A_{t}=$ $\{p\}$. Then

$$
\bigcap_{t \in T}\left(A_{t} \oplus C\right)=\bigcap_{t \in T} A_{t} \oplus C
$$

Lemma 4.7. Let $A \in \mathcal{K}(X)$ and let $x, y \in X$. Then aff $(A-x)=$ aff $A-x$. Moreover, if $x, y \in$ aff $A$, then aff $A-x=\operatorname{aff} A-y$.

Proof. We prove that aff $(A-x)=$ aff $A-x$. If $u \in$ aff $(A-x)$, then there exist $\alpha_{1}, \ldots, \alpha_{n} \in \mathbb{R}$, and $\alpha_{1}+\cdots+\alpha_{n}=1, a_{1}, \ldots, a_{n} \in A$ such that

$$
u=\sum_{i=1}^{n} \alpha_{i}\left(a_{i}-x\right)=\sum_{i=1}^{n} \alpha_{i} a_{i}-x \sum_{i=1}^{n} \alpha_{i}=\sum_{i=1}^{n} \alpha_{i} a_{i}-x \in \text { aff } \mathrm{A}-\mathrm{x} .
$$

Moreover, let $W$ be an affine space and let $p, q, w \in W$. Then $W-p=W-q$. Let $x \in W-p$ so $x=w-p+q-q \in W-q$, hence $W-p \subset W-q$. In a similar way, we obtain $W-q \subset W-p$. Hence $W-q=W-p$ what ends the proof.

Lemma 4.8. Let $\left\{A_{s}\right\}_{s \in S},\left\{B_{t}\right\}_{t \in T}$ are two families of subsets of $X$ such that

(i) aff $A_{s_{1}}=$ aff $A_{s_{2}}, s_{1}, s_{2} \in S$

(ii) aff $A_{s} \cap$ aff $B_{t}=\{p\}, s \in S, t \in T$.

Then

$$
\bigcap_{s \in S, t \in T}\left(A_{s} \oplus B_{t}\right)=\bigcap_{s \in S} A_{s} \oplus \bigcap_{t \in T} B_{t}
$$

Proof. Let $t \in T$. Using the Corollary 4.6 for the $B_{t}$ and $A_{s}$ sets we have

$$
\bigcap_{s \in S}\left(B_{t}+A_{s}\right)=\bigcap_{s \in S} A_{s}+B_{t}
$$

Since aff $\left(\bigcap_{s \in S} A_{s}\right) \subset \bigcap_{s \in S}$ aff $A_{s}=$ aff $\mathrm{A}_{\mathrm{s}}$, then using the previous collorary again for $B_{t}$ and $C=\bigcap_{s \in S} A_{s}$ sets we have $\bigcap_{t \in T}\left(B_{t}+C\right)=\bigcap_{t \in T} B_{t}+C$. By (5) we have $\bigcap_{t \in T} \bigcap_{s \in S}\left(B_{t}+A_{s}\right)=\bigcap_{t \in T}\left(\bigcap_{s \in S} A_{s}+B_{t}\right)=\bigcap_{t \in T}\left(C+B_{t}\right)=$ $\bigcap_{t \in T} B_{t}+C=\bigcap_{t \in T} B_{t}+\bigcap_{s \in S} A_{s}$.

Lemma 4.9. Let $S_{1}, S_{2} \subset X$ be convex sets and aff $S_{1} \cap$ aff $S_{2}=\{p\}$. Moreover let $A \subset$ aff $S_{1} \oplus$ aff $S_{2}$ and let $S=S_{1} \oplus S_{2}$. Then

$$
S \dot{-} A=\left(S_{1} \dot{-} A_{1}\right) \oplus\left(S_{2} \dot{-} A_{2}\right)
$$

where $A_{i}=\pi\left(A\right.$, aff $\left.S_{i}\right), i=0,1$, is a parallel projection onto the subspace aff $S_{i}$. 
Proof. Using the definition $S \stackrel{\bullet}{-} A=\bigcap_{a \in A}(S \stackrel{\bullet}{-} a)$ of Minkowski subtraction we have

$$
S \dot{-} A=\bigcap_{x \in A_{1}, y \in A_{2}}\left(S_{1} \oplus S_{2}-x \oplus y\right)=\bigcap_{x \in A_{1}, y \in A_{2}}\left(\left(S_{1}-x\right) \oplus\left(S_{2}-y\right)\right) .
$$

Putting $A_{x}=S_{1}-x, x \in A_{1}$ and $B_{y}=S_{2}-y, y \in A_{2}$ we have aff $A_{x}=\operatorname{aff}\left(S_{1}-x\right)$ and from the previous lemma, aff $\left(S_{1}-x\right)=\operatorname{aff} S_{1}-x=\operatorname{aff} S_{1}-y=\operatorname{aff}\left(S_{1}-y\right)=$ aff $A_{y}, x, y \in A_{1}$. Similary aff $B_{x}=$ aff $B_{y}$ for every $x, y \in A_{2}$. Moreover because $p \in$ aff $S_{1} \cap$ aff $S_{2}$ so aff $A_{x}=$ aff $\left(S_{1}-x\right)=$ aff $S_{1}-p, x \in A_{1}$, aff $B_{y}=$ aff $S_{2}-p$, $x \in A_{2}$. We have aff $A_{x} \cap$ aff $B_{y}=\left(\right.$ aff $\left.S_{1}-p\right) \cap\left(\right.$ aff $\left.S_{2}-p\right)=\{0\}$. Now, using the Lemma 4.8 and equality (6) we get $\bigcap_{x \in A_{1}, y \in A_{2}}\left(\left(S_{1}-x\right) \oplus\left(S_{2}-y\right)\right)=$ $\bigcap_{x \in A_{1}, y \in A_{2}}\left(A_{x} \oplus A_{y}\right)=\bigcap_{x \in A_{1}} A_{x} \oplus \bigcap_{y \in A_{2}} A_{y}=\bigcap_{x \in A_{1}}\left(S_{1}-x\right) \oplus \bigcap_{y \in A_{2}}\left(S_{2}-\right.$ $y)=\left(S_{1} \dot{-} A_{1}\right) \oplus\left(S_{2} \dot{-} A_{2}\right)$.

Corollary 4.10. Under the assumptions of the above lemma we have

$$
S \dot{-}(S \dot{-} A)=\left(S_{1} \dot{-}\left(S_{1} \dot{-} A_{1}\right)\right) \oplus\left(S_{2} \dot{-}\left(S_{2} \dot{-} A_{2}\right)\right) .
$$

Proof. We have $S-2 p=\left(S_{1}-p\right) \oplus\left(S_{2}-p\right) \subset$ aff $\left(S_{1}-p\right) \oplus$ aff $\left(S_{2}-p\right)$ and $S \dot{-} A=\left(S_{1} \dot{-} A_{1}\right) \oplus\left(S_{2} \dot{-} A_{2}\right), S_{1} \dot{-} A_{1} \subset$ aff $\left(S_{1}-p\right), S_{2} \dot{-} A_{2} \subset$ aff $\left(S_{2}-p\right)$, hence

$$
S \dot{-} A=\left(S_{1} \dot{-} A_{1}\right) \oplus\left(S_{2} \dot{-} A_{2}\right) \subset \text { aff }\left(S_{1}-p\right) \oplus \text { aff }\left(S_{2}-p\right) .
$$

Using Lemma 4.9 we obtain $S \dot{-}(S \dot{-} A)-2 p=(S-2 p)-(S \dot{-} A)=$ $\left[\left(S_{1}-p\right) \oplus\left(S_{2}-p\right)\right] \dot{-}(S \dot{-} A)=\left[\left(S_{1}-p\right) \dot{-} \pi\left(S \dot{-} A\right.\right.$, aff $\left.\left.\left(S_{1}-p\right)\right)\right] \oplus\left[\left(S_{2}-\right.\right.$ $p) \dot{-} \pi\left(S \dot{-} A\right.$, aff $\left.\left.\left(S_{2}-p\right)\right)\right]=\left[\left(S_{1}-p\right) \dot{-}\left(S_{1} \dot{-} A_{1}\right)\right] \oplus\left[\left(S_{1}-p\right) \dot{-}\left(S_{1} \dot{-} A_{1}\right)\right]=$ $\left[S_{1} \dot{-}\left(S_{1} \dot{-} A_{1}\right)\right] \oplus\left[S_{1} \dot{-}\left(S_{1} \dot{-} A_{1}\right)\right]-2 p$. Therefore

$$
S \dot{-}(S \dot{-} A)=\left(S_{1} \dot{-}\left(S_{1} \dot{-} A_{1}\right)\right) \oplus\left(S_{2} \dot{-}\left(S_{2} \dot{-} A_{2}\right)\right) .
$$

Theorem 4.11. If $S_{1}, S_{2} \in \mathcal{S}$ and aff $S_{1} \cap$ aff $S_{2}=\{p\}$, then $S_{1} \oplus S_{2} \in \mathcal{S}$.

Proof. Let $A=\left(S_{1} \oplus S_{2}\right) \dot{-}\left(S_{1} \oplus S_{2} \dot{-} A\right)$. Denote $B_{1}=S_{1} \dot{-}\left(S_{1} \dot{-} A_{1}\right)$ and $B_{2}=S_{2} \dot{-}\left(S_{2} \dot{-} A_{2}\right)$. From previous lemma we know that $A=B_{1} \oplus B_{2}$, where $A_{i}=\pi\left(A\right.$, aff $\left.S_{i}\right)$. Hence $B_{1}=A_{1}, B_{2}=A_{2}$. Because $S_{1}, S_{2} \in \mathcal{S}$ so from condition $S_{1} \dot{-}\left(S_{1} \dot{-} A_{1}\right)=A_{1}$ there exists $T_{1}$ such that $S_{1}=T_{1}+B_{1}$. Similary $S_{2}=T_{2}+B_{2}$. So $S_{1} \oplus S_{2}=\left(B_{1}+T_{1}\right) \oplus\left(B_{2}+T_{2}\right)=\left(B_{1} \oplus B_{2}\right)+\left(T_{1}+T_{2}\right)=A+$ $\left(T_{1}+T_{2}\right)$. Hence $A$ is a summand of the set $S_{1} \oplus S_{2}$. Therefore $S_{1} \oplus S_{2} \in \mathcal{S}$.

\section{Some properties of a class $\mathcal{C}$ of sets}

Now let us define a class $\mathcal{C} \subset \mathcal{K}(X)$ by the following condition: $A \in \mathcal{C}$ if and only if its intersection with any summand of $A$ is still a summand of $A$.

Lemma 5.1. Let $A \in \mathcal{C}$. Then the intersection of any finite number of translates of $A$ is a summand of $A$. 
Proof. Let $n=2$, hence $\left(A-x_{1}\right) \cap\left(A-x_{2}\right)=\left(A-x_{1}+x_{2}\right) \cap A-x_{2}$. We denote

$$
C=\left(A-x_{1}+x_{2}\right) \cap A \text { and } D=C-x_{2} .
$$

Since $C$ is a summand of $A$ so $D$ is a summand of $A$. Let the lemma holds true for $k$ translates, i.e., $\left(A-x_{1}\right) \cap\left(A-x_{2}\right) \cap \cdots \cap\left(A-x_{k}\right) \cap\left(A-x_{k+1}\right)=$ $\left[\left(A-x_{1}\right) \cap\left(A-x_{2}\right) \cap \cdots \cap\left(A-x_{k}\right)+x_{k+1}\right] \cap A-x_{k+1}$. We denote

$$
E=\left[\left(A-x_{1}\right) \cap\left(A-x_{2}\right) \cap \cdots \cap\left(A-x_{k}\right)+x_{k+1}\right] \cap A \quad \text { and } \quad F=E-x_{k+1} .
$$

Hence the sets $E$ and $F$ are summands of $A$.

Lemma 5.2. Let $X$ be a topological vector space and let $A, A_{\lambda} \in \mathcal{K}(X)$ for $\lambda \in \Lambda$. Suppose that the family $\left\{A_{\lambda}\right\}_{\lambda \in \Lambda}$ is a chain of summands of the set $A$, then the set $\bigcap_{\lambda \in \Lambda} A_{\lambda}$ is also a summand of the set $A$.

Proof. It can be proved (see [4, p. 49, Lemma 4.11]) that, if $C, D_{\lambda} \in \mathcal{K}(X)$, and $\left\{D_{\lambda}\right\}_{\lambda \in \Lambda}$ is a chain, then

$$
C+\bigcap_{\lambda \in \Lambda} D_{\lambda}=\bigcap_{\lambda \in \Lambda}\left(C+D_{\lambda}\right) .
$$

Let $A, A_{\lambda} \in \mathcal{K}(X)$ for $\lambda \in \Lambda$ and let the family $\left\{A_{\lambda}\right\}_{\lambda \in \Lambda}$ be a chain of summands of the set $A$. Then there exists $B_{\lambda} \in \mathcal{K}(X)$, such that $A=A_{\lambda}+B_{\lambda}$ for $\lambda \in \Lambda$. Since $B_{\lambda}=A \dot{-} A_{\lambda} \subset A \dot{-}\left(\bigcap_{\lambda \in \Lambda} A_{\lambda}\right)$, we deduce that the set $\overline{\bigcup_{\lambda \in \Lambda} B_{\lambda}}$ is compact, and since the family $\left\{B_{\lambda}\right\}_{\lambda \in \Lambda}$ is a chain it is convex. Using the equality (7) we obtain

$$
A \subset \bigcap_{\lambda \in \Lambda}\left(A_{\lambda}+\overline{\bigcup_{\lambda \in \Lambda} B_{\lambda}}\right)=\bigcap_{\lambda \in \Lambda} A_{\lambda}+\overline{\bigcup_{\lambda \in \Lambda} B_{\lambda}}
$$

On the other hand

$$
\bigcap_{\lambda \in \Lambda} A_{\lambda}+\overline{\bigcup_{\lambda \in \Lambda} B_{\lambda}} \subset \overline{\bigcap_{\lambda \in \Lambda} A_{\lambda}+\bigcup_{\lambda \in \Lambda} B_{\lambda}} \subset \overline{\bigcup_{\lambda \in \Lambda}\left(A_{\lambda}+B_{\lambda}\right)}=\bar{A}=A .
$$

Therefore $A=\bigcap_{\lambda \in \Lambda} A_{\lambda}+\overline{\bigcup_{\lambda \in \Lambda} B_{\lambda}}$ and the set $\bigcap_{\lambda \in \Lambda} A_{\lambda}$ is a summand of the set $A$.

Example 5.3. Let $X=c_{0}$ be the real Banach space of all sequences convergent to 0 with the supremum norm, $\|x\|=\sup _{k}\left|x_{k}\right|$. Let $A=\left\{x \in c_{0}:\|x\| \leq 1\right\}$ be the unit ball, $A_{m}=\left\{x \in A: x_{1}=\cdots=x_{m}=1\right\}, C_{m}=\left\{x \in A: x_{k}=0\right.$ for $k>m\}, b_{m}=(-1, \ldots,-1,0, \ldots)$, where the first $m$ components equals -1 . Let $B_{m}=b_{m}+C_{m}$ for $m \in \mathbb{N}$. Then $A, A_{m}, B_{m} \in \mathcal{B}(X), A_{m}+B_{m}=A$ and $A_{m+1} \subset A_{m}$ for $m \in \mathbb{N}$. Hence the family $\left\{A_{m}\right\}_{m \in \mathbb{N}}$ is a chain of summands of the set $A$, but the set $\bigcap_{m \in N} A_{m}$ is empty and is obviously not a summand of $A$. This example shows that the assumption $A \in \mathcal{K}(X)$ in Lemma 5.2 is essential. 
Lemma 5.4. Let $X$ be a normed vector space, $B \in \mathcal{B}(X)$ and let $\left\{b_{n}\right\} \subset B$ be a dense set in $B$. Then $\bigcap_{x \in B}(A-x)=\bigcap_{n=1}^{\infty}\left(A-b_{n}\right)$ for any $A \in \mathcal{B}(X)$.

Proof. It is enough to show that $\bigcap_{n=1}^{\infty}\left(A-b_{n}\right) \subset \bigcap_{x \in B}(A-x)$. Let $z \in$ $\bigcap_{n=1}^{\infty}\left(A-b_{n}\right)$ and let $x_{0} \in B$. Then there exists a subsequence of $\left(b_{n}\right)$, which we denote by $\left(b_{n_{k}}\right)$ such that $b_{n_{k}} \rightarrow x_{0}$. We have $z=a_{n_{k}}-b_{n_{k}}, a_{n_{k}} \in A$. The closedness of $A$ and the equality $\lim _{k \rightarrow \infty} a_{n_{k}}=z+x_{0} \in A$ imply $z \in A-x_{0}$.

Theorem 5.5. Let $A$ be a convex, compact subset of a normed, separable space $X$ and let $A \in \mathcal{C}$. Then for any set $B \in \mathcal{K}(X)$ set $A-B$ is a summand of $A$.

Proof. From Lemma 5.4 we have $A \dot{-} B=\bigcap_{n=1}^{\infty}\left(A-b_{n}\right)$, where $\left\{\bar{b}_{n}\right\}=B$. Now from Lemmas 5.2 and 5.4 and equality $\bigcap_{n=1}^{\infty}\left(A-b_{n}\right)=\bigcap_{k=1}^{\infty}\left(\bigcap_{n=1}^{\infty}\left(A-b_{n}\right)\right)$, we obtain that $A-B$ is a summand of $A$.

Proposition 5.6. The cubes and simplexes belong to the family $\mathrm{C}$.

Proof. Let $C, D \in \mathcal{K}(X)$ and $C+D=\left[a_{1}, b_{1}\right] \times \cdots \times\left[a_{n}, b_{n}\right]$. Let $p_{i}: \mathbb{R}^{n} \rightarrow \mathbb{R}$ and $p_{i}(x)=x_{i}$. Then $p_{k}(C+D)=\left[a_{k}, b_{k}\right]$ and hence $p_{k}(C)+p_{k}(D)=\left[a_{k}, b_{k}\right]$. Let $C_{1}=p_{1}(C) \times \cdots \times p_{n}(C)$ and $D_{1}=p_{1}(D) \times \cdots \times p_{n}(D)$. Then $C \subset C_{1}, D \subset D_{1}$. We have

$$
C+D_{1} \subset C_{1}+D_{1} \subset\left[a_{1}, b_{1}\right] \times \cdots \times\left[a_{n}, b_{n}\right]=C+D,
$$

and by the cancellation law we obtain $D_{1} \subset D$. Similary $C_{1} \subset C$. Therefore $C=C_{1}, D=D_{1}$. We just proved that every summand of cube is still a cube. Hence the intersection of cube with any summand of cube is still a summand of cube. Therefore, cubes belongs to $\mathcal{C}$.

From the indecomposability of a simplex it follows that any summand of a simplex $S$ is a simplex homothetic to $S$. Hence the intersection $S$ with any summand of $S$ is still a summand of $S$. Therefore, simplexes belongs to $\mathcal{C}$.

There is still the open question how to characterise the class $\mathcal{C}$ for the space $\mathbb{R}^{n}(n \geq 2)$.

\section{References}

[1] Eggleston, H. G., Convexity. Cambridge: Cambridge Univ. Press 1958.

[2] Grzybowski, J., Przybycien, H. and Urbański, R., On summands of closed bounded convex sets. J. Anal. Appl. 21 (2002)(4), 845 - 850.

[3] Kaczmarek, S., Minimal pairs in classes of frustums. Comm. Math. 39 (1999), $74-76$.

[4] Pallaschke, D. and Urbański, R., Pairs of Compact Convex Sets. Fractional Arithmetic with Convex Sets. Math. Appl. 548. Dortrecht: Kluwer 2002. 
[5] Sallee, G. T., Pairs of sets of constant relative width. J. Geom. 29 (1987)(1), $1-11$.

[6] Sallee, G. T., Realeaux polytopes. Mathematika 17 (1970), 315 - 323.

[7] Schneider, R., Convex Bodies: The Brunn-Minkowski Theory. Encykl. Math. Appl. 44. Cambridge: Cambridge Univ. Press 1993.

[8] Yaglom, I. M. and Boltjanskiü, V. G., Convex Figures (Engl. transl.). New York: Holt, Rinehart and Winston 1960.

Received April 19, 2005; revised December 3, 2005 